\title{
Radiation Pressure from Interstellar Hydrogen Observed by IBEX through Solar Cycle 24
}

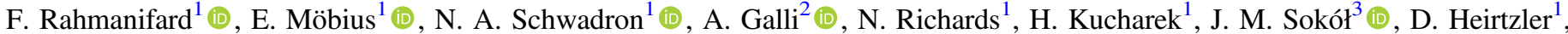

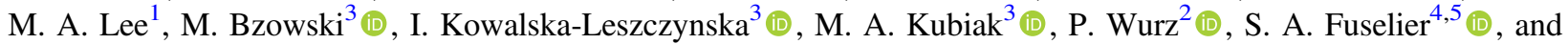 \\ D. J. McComas ${ }^{6}$ (iD \\ ${ }^{1}$ Physics Department, Space Science Center, University of New Hampshire, Durham, NH 03824, USA; F.Rahmanifard@unh.edu \\ ${ }^{2}$ Physics Institute, University of Bern, Bern, 3012, Switzerland \\ ${ }^{3}$ Space Research Centre, Polish Academy of Sciences, Warsaw, 00-716, Poland \\ ${ }^{4}$ Southwest Research Institute, San Antonio, TX 78228, USA \\ ${ }^{5}$ University of Texas at San Antonio, San Antonio, TX 78228, USA \\ ${ }^{6}$ Department of Astrophysical Sciences, Princeton University, Princeton, NJ 08544, USA \\ Received 2019 April 29; revised 2019 November 14; accepted 2019 November 17; published 2019 December 20
}

\begin{abstract}
As the Sun moves through the local interstellar medium (LISM), neutral atoms travel through the heliosphere and can be detected by IBEX. We consider interstellar neutral (ISN) hydrogen atoms with a drifting Maxwellian distribution function in the LISM that travel on almost hyperbolic trajectories to the inner heliosphere. They are subject to solar gravity and radiation pressure, as well as ionization processes. For ISN H, the radiation pressure, which exerts an effective force comparable to gravitation, decelerates individual atoms and shifts the longitude of their observed peak relative to that of ISN He. We used the peak longitude of the observed flux in the lowest energy channel of $I B E X$-Lo to investigate how radiation pressure shifts the ISN H signal over almost an entire solar cycle (2009-2018). Thus, we have created a new methodology to determine the Ly $\alpha$ effective radiation pressure from IBEX ISN $\mathrm{H}$ data. The resulting effective ratio of the solar radiation pressure and gravitation $\left(\mu_{\text {eff }}=1.074 \pm 0.038\right)$, averaged over cycle 24 , appears to agree within the uncertainties with simulations based on total irradiance observations ${ }^{7}$ while being higher by $\sim 21 \%$. Our analysis indicates an increase of $\mu_{\text {eff }}$ with solar activity, albeit with substantial uncertainties. Further study of IBEX H response functions and future Interstellar Mapping and Acceleration Probe data should provide significant reduction of the uncertainties and improvements in our understanding of the effects of radiation pressure on ISN atoms.
\end{abstract}

Unified Astronomy Thesaurus concepts: Solar activity (1475); Solar wind (1534); Heliosphere (711); Interstellar atomic gas (833); Interstellar medium (847); Space plasmas (1544)

\section{Introduction}

The very local interstellar medium (VLISM) is a dilute, partially ionized gas consisting of neutral atoms, such as $\mathrm{H}, \mathrm{He}$, $\mathrm{C}, \mathrm{O}$, and $\mathrm{Ne}$, and a plasma part consisting of electrons, protons, and heavier ions. The relative motion of the Sun with respect to the VLISM at a speed of $\sim 25 \mathrm{~km} \mathrm{~s}^{-1}$, reported by several studies, causes an inflow of the interstellar neutral (ISN) atoms into the inner heliosphere, unimpeded by the solar magnetic field. However, the distribution function of these ISN atoms is modified by the Sun's gravity and, for $\mathrm{H}$ atoms, radiation pressure as well. Also, ISN atoms are depleted via ionization processes both at the heliospheric interface and after they enter the heliosphere.

The $\sim 25 \mathrm{~km} \mathrm{~s}^{-1}$ inflow speed mentioned earlier is obtained by direct neutral gas observations with Ulysses (Witte 2004; Bzowski et al. 2014; Wood et al. 2015), UV backscattering observations from the Solar and Heliospheric Observatory (SOHO; Vallerga et al. 2004), and neutral gas observations with the Interstellar Boundary Explorer (IBEX; Fuselier et al. 2009; McComas et al. 2009) for ISN He (e.g., Bzowski et al. 2015; McComas et al. 2015; Schwadron et al. 2015). Other studies investigated ISN He parameters, including the temperature, density, and flow direction (Lallement \& Bertin 1992; Linsky et al. 1993; Gloeckler et al. 2004; Möbius et al. 2004; the latter study provides a thorough discussion of methods to

Kowalska-Leszczynska et al. (2018b). investigate VLISM parameters using ISN He). A similar set of studies was conducted to find the parameters associated with ISN $\mathrm{H}$. The density of ISN $\mathrm{H}$ at the termination shock needed to slow down the solar wind from the values measured by Ulysses at $\sim 5$ au to the values reported by Voyager upstream of the termination shock was estimated as $0.09 \mathrm{~cm}^{-3}$ (Richardson et al. 2008). Based on the pickup ion production rate observed by Ulysses (Gloeckler \& Geiss 2001), the density of ISN H at the termination shock was estimated to be $\sim 0.087 \mathrm{~cm}^{-3}$ (Bzowski et al. 2008). Another study based on the Ly $\alpha$ radiation transport using Cassini and Voyager 1 data reported values between 0.085 and $0.095 \mathrm{~cm}^{-3}$ for this parameter (Pryor et al. 2008). The Ly $\alpha$ absorption line and its reemission by ISN $\mathrm{H}$, known as helioglow, measured by $\mathrm{SOHO} / \mathrm{SWAN}$ was used to find the flow direction of ISN H (Lallement et al. 2005, 2010). Müller et al. (2008) used various global heliosphere models to show that the termination shock location agrees between these models within reasonable errors.

Far away from the heliospheric interface, the ionized and neutral parts of the VLISM are highly coupled through charge exchange and elastic collisions. Once the interstellar plasma meets the obstacle created by the heliosphere, it is diverted, decelerated (possibly through a bow wave rather than a bow shock; see McComas et al. 2012; Zank et al. 2013), and heated. Along with this interaction, the VLISM magnetic field is draped around the heliosphere. On the other hand, the neutral part of the VLISM, not affected by the magnetic field of the heliosphere, 
moves toward the Sun without substantial changes. In the outer heliosheath, i.e., the space between the bow shock (or bow wave) and the heliopause, charge exchange continues, and two new ion and neutral populations are created as a result. The former neutral particles become ionized and start interacting with the plasma part of the VLISM, which causes them to be deflected and thermalized (Frisch et al. 2009). Protons of the outer heliosheath are neutralized and decouple from the ISM magnetic field while maintaining their kinematic properties before the collision to create a new population: the so-called "secondary population," as opposed to the "primary population." This secondary population is hotter and slower than the primary flow and piles up to form the hydrogen wall (for a more detailed discussion, see Frisch et al. 2009). In turn, the creation of the secondary population leads to selective filtration of the primary population, which leaves a primary distribution that is faster and cooler. A 3D kinetic model of the heliospheric interface showed an $\sim 10 \%$ increase in the velocity of primary $\mathrm{H}$ atoms and a less than $8 \%$ decrease in their temperature toward the termination shock (Izmodenov \& Baranov 2006).

The ionization processes that occur further inside the heliopause, including photoionization, charge exchange, and electron impact ionization, lead to further loss of the neutral particles (e.g., Bzowski et al. 2013; Bochsler et al. 2014). For the case of ISN H, charge exchange and photoionization are the dominant processes. Inside the heliosphere, both scale with $1 / r^{2}$, since the charge exchange is proportional to the solar wind flux and photoionization scales with the solar UV photon intensity. While the radiation force decelerates the ISN H flow, these loss processes reduce the flow. As a result, the ISN H density decreases, and its energy does not increase as much or at all as it approaches $1 \mathrm{au}$, where we observe it with the IBEXLo neutral atom camera. An important selection effect due to ionization inside the heliosphere causes faster atoms to be more likely to survive to 1 au (Bzowski et al. 1997).

The low flux of ISN H is not the only barrier in detecting the signal with IBEX. After passing the IBEX-Lo collimator, the incident neutral atoms hit a diamond-like carbon surface that is coated with a monolayer of water. Here they either are converted to or sputter negative ions. The conversion surface, which is key to detecting the ISN neutral atoms (Wurz et al. 1997, 2008; Fuselier et al. 2009), presents a challenge when it comes to separating ISN $\mathrm{H}$ from $\mathrm{He}$ atoms. While the lowenergy ISN $\mathrm{H}$ atoms are expected to be converted at the surface to $\mathrm{H}^{-}$ions and form a broad but faint signal in the first two energy steps, the much more abundant and energetic ISN He atoms sputter $\mathrm{H}^{-}$ions from the conversion surface that flood the first four energy steps (see Figure 1 from Möbius et al. 2012). As a result, it is extremely challenging to extract the ISN $\mathrm{H}$ signal from the more abundant ISN He, specifically during years of high solar activity when the $\mathrm{H}$ signal drops off dramatically. The radiation force, however, shifts the peak longitude of the ISN H signal so it does not coincide with other neutral atom peaks, making ISN $\mathrm{H}$ detectable in spite of the interference by the sputter products of ISN He.

The first direct detection of ISN H was reported by Möbius et al. (2009). Saul et al. (2012) provided a first quantitative analysis of the first $2 \mathrm{yr}$ of $I B E X$ observations, revealing an offset in the signal in both longitude and latitude over time. They developed two techniques to distinguish the ISN H signal from the sputtered ISN He: (1) determine and subtract ISN He by modeling the observed spectrum as the sum of ISN He and the residual ISN H component (the so-called "subtraction method") and (2) use the He signal from a higher energy step as a proxy to extract the ISN H signal. The resulting ISN H signal was shown to be consistent with a radiation pressure that exceeds the gravitational force. It is noteworthy that this is even the case for 2009-2010, i.e., during low solar activity. As the solar activity rose, the ISN H signal was found to fall near background levels in 2012 (Saul et al. 2013). Saul et al. (2013) also reported an increasing longitudinal offset with increasing solar activity in the first $4 \mathrm{yr}$ of IBEX observations. During the most active phases of cycle 24, the signal almost disappeared, partially due to switching to a lower post-acceleration voltage in the summer of 2012, which resulted in even lower counting statistics (McComas et al. 2014). The most recent IBEX observations of ISN $\mathrm{H}$ showed signal recovery in the consecutive seasons of 2017-2018 as we approached another solar minimum (Galli et al. 2019). The longitude offset of the ISN H signal peak appeared to be overshadowed by the uncertainties associated with the strong ISN He signal in their analysis.

Early attempts to model ISN atoms were inspired by the backscattered solar Ly $\alpha$ measurements resulting in the socalled cold gas models (Fahr 1968; Blum \& Fahr 1970; Axford 1972; Vasyliunas \& Siscoe 1976). These models assume that the distribution function of the ISN gas has a central velocity and zero temperature. The cold gas model was generalized to include the gravitational force and ionization processes. The next step in modeling the ISN gas allowed consideration of a drifting Maxwellian distribution at infinity with a finite temperature. A class of hot gas models also took into account solar gravitation and radiation pressure, as well as ionization losses (Fahr 1971, 1979; Meier 1977; Thomas 1978; Wu \& Judge 1979). While classical hot gas models adequately predict general estimates of ISN H flow, additional improvements consider important effects: (1) modification of the interstellar gas distribution when entering the heliosphere through the interface region (Baranov \& Malama 1993; Izmodenov 2001; Izmodenov \& Alexashov 2015; Katushkina et al. 2015), (2) latitudinal asymmetries in solar parameters (Bzowski et al. 2002; Mccomas et al. 2003; McComas et al. 2006, 2008), and (3) solar cycle variability (Bzowski \& Rucinski 1995; Bzowski et al. 1997, 2003, 2008; Pryor et al. 2003; Izmodenov 2004; Tarnopolski \& Bzowski 2009).

In this work, we concentrate on a quantitative determination of the effect of radiation pressure on ISN $\mathrm{H}$ and the longitudinal shift of the ISN $\mathrm{H}$ peak over almost a full solar cycle. A reported discrepancy in the ratio of ISN H counts in the lowest two energy channels between all available models and the observed signals (further explained in Section 7) compelled us to focus our study of the longitudinal shift of ISN $\mathrm{H}$ on the lowest energy step. We fit a Gaussian to the peak of the ISN H signal observed by IBEX (Galli et al. 2019) for each season to find its longitude. Using the analytical full integration model (aFINM; further explained in Section 3), we find an effective parameter $\mu$ for each season to match the observed peak longitude. We aim to show variations in the peak longitude and, as a result, variations in parameter $\mu$ with solar activity. We briefly discuss the basis for the existing models in Section 2, with a focus on the trajectories of the ISN H atoms. In Section 3, the resulting distribution function and its essential parameters are reviewed, along with a brief history of the current models that are specialized to predict the ISN flux observed by $I B E X$. In Section 4, we describe the IBEX-Lo 


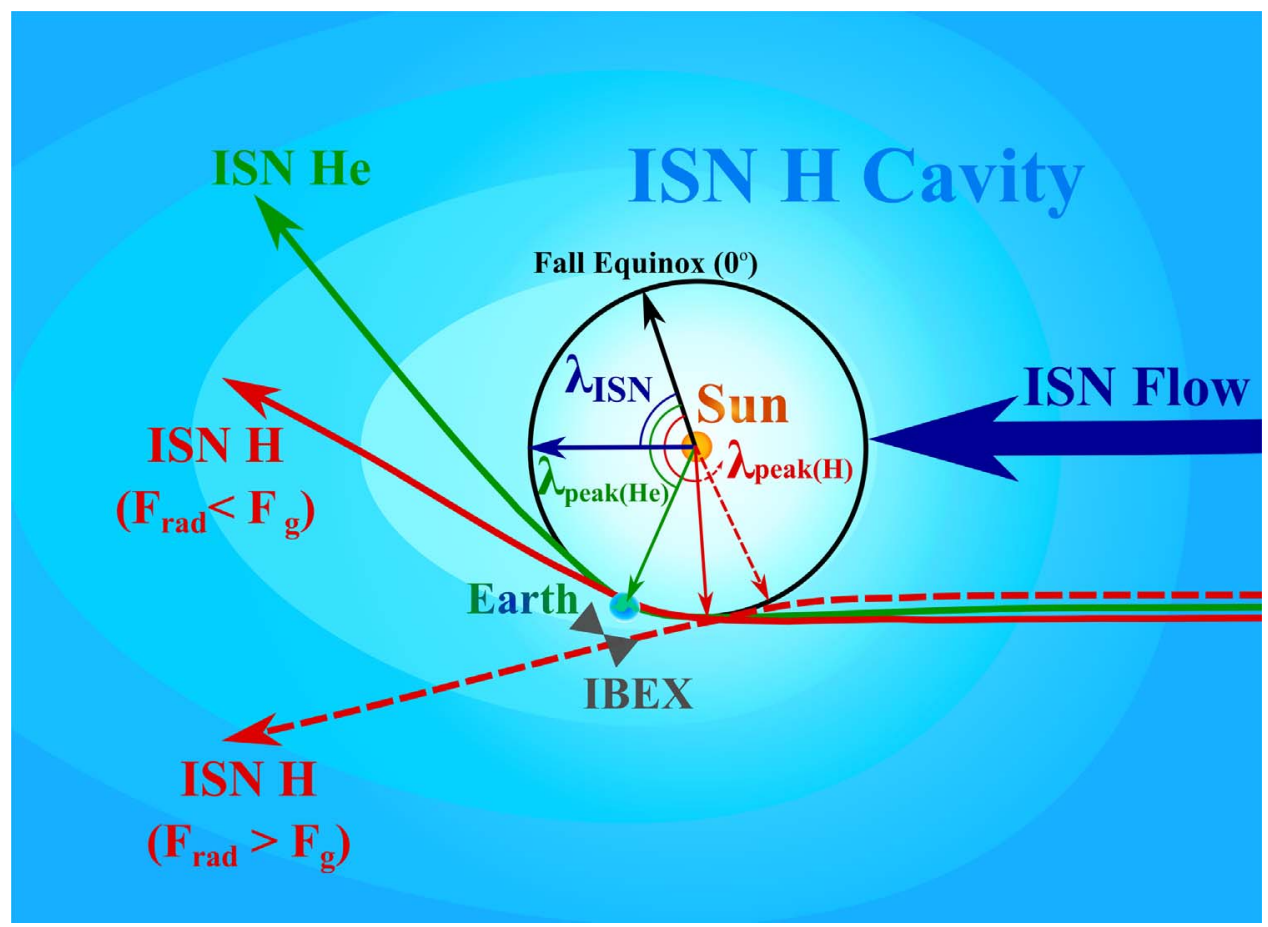

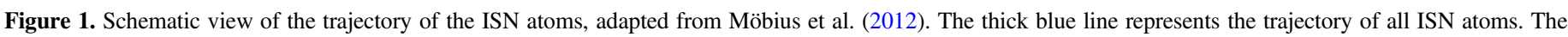

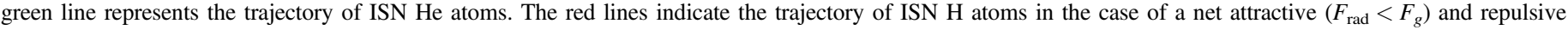
$\left(F_{\text {rad }}>F_{g}\right)$ force.

detector and its energy channel response to the ISN H signal. The data used in this paper and their retrieval methods are presented in Section 5. The observed temporal shift in the ISN $\mathrm{H}$ signal and the predicted parameter $\mu$ based on that shift is presented in Section 6 and discussed in Section 7. The justification for the use of a stationary model in the complex case of ISN $\mathrm{H}$ is relegated to the Appendix. Concluding remarks are provided in Section 8.

\section{ISN H Trajectory}

The ISN atoms have the same bulk speed, and their mean energy scales with their mass. The Sun's gravitational field and ionization processes modify the distribution function of the ISN gas as it flows through the heliosphere and lead to characteristic trajectories for the neutral atoms. Figure 1 shows a schematic diagram of the trajectory of the ISN atoms. For ISN He, Ne, and $\mathrm{O}$, the attractive gravitational field bends the trajectories toward the Sun (green line in Figure 1). For ISN H, this characteristic trajectory is further modified by radiation pressure and results in a possibly repulsive net force $\left(F_{\text {net }}\right)$ if $F_{\text {rad }}>F_{g}$, which bends the trajectory away from the Sun,

$$
F_{\text {net }}=F_{g}+F_{\text {rad }}=(1-\mu) F_{g},
$$

where $\mu$ is a dimensionless parameter describing the ratio of the radiation pressure force to the gravitational force $\left(\mu=\left|F_{\text {rad }}\right| /\left|F_{g}\right|\right)$, which is a function of time, heliolatitude, and radial velocity.

As shown in Figure 1, a repulsive net force results in ISN H atom trajectories bent outward from the Sun that reach their perihelion at larger ecliptic longitude and thus are observed by $I B E X$ in later orbits, increasing the peak longitude $\left(\lambda_{\text {peak }}\right)$. An attractive net force in this case $\left(F_{\text {rad }}<F_{g}\right)$ moves the trajectory's perihelion toward earlier IBEX orbits, decreasing $\lambda_{\text {peak }}$, although still at larger values than found for ISN He and $\mathrm{O}$ due to a weaker net force acting on ISN $\mathrm{H}$.

\section{ISN H Distribution Function}

Modeling ISN atoms observed by IBEX has played a major role in understanding the nature of the VLISM and how it interacts with the heliosphere. An analytical model of the interstellar gas was presented by Lee et al. $(2012,2015)$ that was basically the classical hot model tailored to model IBEX observations. Lee et al. (2012) developed an analytic model for the ISN flow based on the invariance of the distribution function along hyperbolic trajectories, assuming a stationary and axisymmetric flow. Assuming constant radiation pressure and ionization rates allows the use of the analytic relations in this model with some modification.

An attempt to combine the Lee et al. (2012) work with numerical methods to simulate ISN flow observed by IBEX-Lo led to the aFINM. In this model, constant radiation pressure and ionization loss rates are assumed to use the analytic relations presented by Lee et al. (2012). However, it uses numerical methods to integrate over the instrument energy steps (using an empirical energy response function), collimator (using the collimator response function), and spin sector. Schwadron et al. (2013) developed aFINM to predict the ISN H count rates observed by IBEX. They performed a $\chi^{2}$ analysis to find $\mu$, as well as the characteristic parameters of the ISN $\mathrm{H}$ bulk flow (vector velocity and temperature) for the years 2009-2011. Schwadron et al. $(2015,2016 a)$ modified the same model to successfully predict count rates for $\mathrm{He}$ and $\mathrm{O}$, and they obtained consistent bulk flow parameters for these ISN species.

The Warsaw Test Particle Model (WTPM) was developed to simulate ISN He (Bzowski et al. 2012) and used to determine that a secondary population of the ISN He is necessary to fully account for the observations. The WTPM was optimized and adjusted to predict the flux of interstellar atoms observed by $I B E X$-Lo considering both primary and secondary populations 
Table 1

Parameters Associated with the ISN H Distribution Function (Identical to Those Used by Kowalska-Leszczynska et al. 2018a)

\begin{tabular}{lcc}
\hline \hline & Primary & Secondary \\
\hline$n_{i}$ & $0.030 \mathrm{~cm}^{-3}$ & $0.054 \mathrm{~cm}^{-3}$ \\
$T_{i}$ & $7443 \mathrm{~K}$ & $16300 \mathrm{~K}$ \\
$V_{i}$ & $25.784^{\mathrm{a}} \mathrm{km} \mathrm{s}^{-1}$ & $18.744 \mathrm{~km} \mathrm{~s}^{-1}$ \\
$\lambda_{i}$ & $75^{\circ} .745$ & 71.57 \\
$\phi_{i}$ & 5.169 & 11.95 \\
\hline
\end{tabular}

Note.

a Considering a selection effect in the outer heliosheath by applying an $\sim 10 \%$ increase in speed and $\sim 8 \%$ decrease in temperature of the primary ISN $\mathrm{H}$ atoms to these values does not significantly change our final results.

for the ISN He (Bzowski et al. 2015; Sokół et al. 2015; Swaczyna et al. 2015). The WTPM was subsequently adapted to simulate the IBEX ISN $\mathrm{H}$ signal as a superposition of contributing signals from the primary and secondary populations of ISN H (Kowalska-Leszczynska et al. 2018a; Galli et al. 2019). A combination of a global kinetic-MHD model of the heliospheric interface and a 3D time-dependent kinetic model (based on the classical hot model) of the ISN $\mathrm{H}$ distribution (Izmodenov \& Alexashov 2015) inside the heliosphere was used to find the ISN $\mathrm{H}$ flux at 1 au observed by IBEX (Katushkina et al. 2015).

Lee et al. (2012) only considered the primary population, although the model can be expanded to secondary populations in a straightforward way, assuming they are represented by a Maxwellian distribution function. In this study, we used aFINM (with some modifications) for a distribution function consisting of two Maxwellian functions associated with the primary and secondary $\mathrm{H}$ populations at the solar wind termination shock,

$$
\begin{gathered}
f=\sum_{i=\operatorname{prim}, \sec } f_{i}, \\
f_{i}=n_{i}\left(2 \pi k_{B} T_{i} / m\right)^{-3 / 2} \times \exp \left(-\left|V-V_{i}\right|^{2}\left(2 k_{B} T_{i} / m\right)^{-1}\right),
\end{gathered}
$$

where $n_{i}, T_{i}$, and $V_{i}$ are the ISN $\mathrm{H}$ density, temperature, and bulk flow velocity for the primary or secondary population at the termination shock. The bulk flow parameters for the primary population (see Table 1) were adopted from Bzowski et al. (2015) based on analysis of IBEX observations of the ISN He with the WTPM. They obtained these values for the velocity and temperature of the ISN He bulk flow parameters at the heliospheric interface, which was adopted here also for the primary ISN H. The ISN H densities for both the primary and secondary populations were taken to be identical to those obtained by Bzowski et al. (2008), as well as the temperature and speed for the secondary population. The flow direction (longitude, $\lambda$, and latitude, $\phi$ ) of the secondary $\mathrm{H}$, however, was adopted from Kubiak et al. (2016), which they found for the ISN He secondary population (for further details, see Kowalska-Leszczynska et al. 2018a). Although in a previous study, Kubiak et al. (2014) had found the secondary ISN He atoms to deflect much more than the secondary $\mathrm{H}$ atoms, Kubiak et al. (2016) demonstrated a less significant deflection for the secondary He atoms. Furthermore, they showed that the inflow direction of the ISN H (Lallement et al. 2010) falls right between the ISN He primary and secondary populations. Since the Lallement et al. (2010) analysis of the SWAN/SOHO observations of the heliospheric $\mathrm{H}$ backscatter glow corresponds to the combined flow of the primary and secondary ISN $\mathrm{H}$ population, this probably suggests that the primary and secondary ISN $\mathrm{H}$ inflow directions might be very similar to those of ISN He.

In this study, we considered charge exchange and photoionization, which are the most dominant processes for the ISN H flow (Bzowski et al. 2013), inside the heliosphere. Although charge exchange and photoionization vary over time, we applied values averaged over the last part of the trajectory for which the ionization rate was above $\sim 10 \%$ of its values at 1 au (roughly the last seven Carrington cycles). Moreover, the charge exchange rate varies with heliolatitude due to the latitudinal variations of the solar wind speed and density (Bzowski et al. 2002). We used the Lindsay \& Stebbings (2005) formula for the charge exchange cross section (for further details, see Bzowski et al. 2013). We determined the charge exchange rate using temporally averaged values of the solar wind speed and density in the heliolatitude associated with the trajectory of the incoming atoms, based on Sokół et al. (2013). Temporally averaged photoionization rates for $\mathrm{H}$ atoms were obtained from a model described in Bzowski et al. (2013). The total ionization rate $\beta$ combines the average charge exchange rate $\left(\beta_{\text {chex,1 au }}(\theta)\right)$ and photoionization rate $\left(\beta_{\mathrm{ph}, 1 \mathrm{au}}\right)$ as a function of the distance from the Sun $(r)$ and heliolatitude $(\theta)$ :

$$
\beta=\left(\beta_{\text {chex }, 1 \mathrm{au}}(\theta)+\beta_{\mathrm{ph}, 1 \mathrm{au}}\right)\left(\frac{r_{E}}{r}\right)^{2} .
$$

Most of the modifications to aFINM in this work reduce computation times. However, more importantly, we improved the averaging methods to take into account temporal and heliolatitudinal variations of the solar wind speed and density, as well as temporal variations of the photoionization. Additionally, we developed a separate numerical tool to justify using a stationary model for the ISN H problem (see the Appendix).

\section{Instrument Description}

The IBEX-Lo instrument accepts neutral atoms from $\sim 10 \mathrm{eV}$ to $2 \mathrm{keV}$ (Fuselier et al. 2009). The incoming particles pass through a collimator (with a FWHM of $7^{\circ}$ ) and then hit a conversion surface, where they are converted to negative ions and/or produce sputtered negative ions. Then they are selected based on their energy per charge by an electrostatic analyzer with eight logarithmically spaced energy steps and accelerated by a post-acceleration voltage. The converted ISN $\mathrm{H}$ ions are predominantly observed in energy steps (e-steps) $1(15 \mathrm{eV}$ center energy) and 2 ( $29 \mathrm{eV}$ center energy), while the ISN He sputter products are distributed over the first four e-steps (Möbius et al. 2012, Figure 1), with similar detection efficiencies in e-steps 1-3 and starkly reduced in e-step 4. The energy response function for each of the energy steps for different species is obtained empirically as a normalized transmission function, $T(E)$, based on its central energy $\left(E_{c}\right)$ and a FWHM $\Delta E / E=0.7$ (Fuselier et al. 2009), resulting in respective maximum and minimum energies $\left(E_{\max }\right.$ and $\left.E_{\min }\right)$, multiplied by a constant geometric factor for each e-step and 
Table 2

H Transmission Function Essential Parameters for E-steps 1 and 2

\begin{tabular}{lccccc}
\hline \hline E-step & $\begin{array}{c}E_{c} \\
(\mathrm{eV})\end{array}$ & $\begin{array}{c}E_{\min } \\
(\mathrm{eV})\end{array}$ & $\begin{array}{c}E_{\max } \\
(\mathrm{eV})\end{array}$ & $\begin{array}{c}G\left(\mathrm{~cm}^{2} \mathrm{sr} \mathrm{keV} / \mathrm{keV}\right) \\
\text { before PAC Voltage Change }\end{array}$ & $\begin{array}{c}G\left(\mathrm{~cm}^{2} \mathrm{sr} \mathrm{keV} / \mathrm{keV}\right) \\
\text { after PAC Voltage Change }\end{array}$ \\
\hline 1 & 15 & 11 & 21 & $7.29 \times 10^{-6} \times 0.93$ & $7.29 \times 10^{-6} \times 0.435$ \\
2 & 29 & 41 & $1.414 \times 10^{-5} \times 0.93$ & $1.414 \times 10^{-5} \times 0.435$ \\
\hline
\end{tabular}

species:

$$
T(E)= \begin{cases}\exp \left(-4 \ln 2 \frac{\left(E / E_{c}-1\right)^{2}}{\Delta_{1}^{2}}\right), & \text { for } E \leqslant E_{c} \\ \exp \left(-4 \ln 2 \frac{\left(E_{c} / E-1\right)^{2}}{\Delta_{2}^{2}}\right), & \text { for } E>E_{c} .\end{cases}
$$

This energy transmission function is from Schwadron et al. (2013), where $\Delta_{1}=2\left(1-E_{\min } / E_{c}\right)$ and $\Delta_{2}=2\left(1-E_{c} /\right.$ $\left.E_{\max }\right)$. They fitted a Gaussian function to laboratory calibration data (see Schwadron et al. 2013; Park et al. 2016). The geometric factors are obtained by multiplying the absolute geometric factor, the energy resolution of the energy steps, and the conversion efficiencies (see Schwadron et al. 2009; Park et al. 2016). For $\mathrm{H}$ atoms, the geometric factor of e-step 2 is almost twice that of e-step 1 (see Table 2), leading to higher count rates predicted for e-step 2 than e-step 1 (at least in model predictions for the expected range of the parameter $\mu$ ). These values are obtained based on calibration data for a neutral hydrogen beam in the laboratory.

Figure 2 shows the energy range of the ISN $\mathrm{H}$ distribution at 1 au (for $\mu=0.9$ and 1.1), along with the normalized transmission functions of the first two energy steps. Since the distribution peak of ISN $\mathrm{H}$ falls between the two energy channels, its signal is very sensitive to the variation of solar parameters and calibration coefficients of the instrument.

\section{Data Selection}

To separate ISN H from He, Schwadron et al. (2013) applied a subtraction method by using the ratios of the count rates for e-step 1/e-step 3 and e-step 2/e-step 3 at the peak of the primary interstellar He (ISN He) flow. They assumed that the same fraction is still valid during later orbits, where the ISN H flow is dominant (and secondary $\mathrm{He}$ is still present), to subtract the sputtered $\mathrm{H}^{-}$ions produced by the ISN He flow. To obtain more reliable H counts, Galli et al. (2019) used a bootstrap method to calculate the ISN He intensity from the $\mathrm{H}^{-}$count rate in e-step 3 for each map pixel, based on the rationale that ISN H (with energies below $40 \mathrm{eV}$ ) does not contribute to the count rates from e-step 3. Then they ascribed the excess $\mathrm{H}^{-}$count rates registered in the first two energy steps to ISN H. For the conversion between the He intensity and the $\mathrm{H}^{-}$count rates, they used conversion factors from laboratory calibration (the so-called "H3-lab" approach), as well as factors derived from in-flight observations (the "H3-inflight" approach). In addition, they applied another bootstrap method ("H2O2-inflight"), where they calculated the ISN $\mathrm{H}$ intensity in the first two energy steps by using empirical $\mathrm{H}^{-} / \mathrm{O}^{-}$ratios for sputtered $\mathrm{H}^{-}$versus sputtered $\mathrm{O}^{-}$count rates in each energy step. Lowering the postacceleration voltage in 2012 resulted in lower sensitivity to $\mathrm{O}$. As a result, the $\mathrm{H} 2 \mathrm{O} 2$ method can only cover the years 2009-2012, for which $\mathrm{O}^{-}$maps are available. These three methods were implemented by Galli et al. (2019) and confirmed

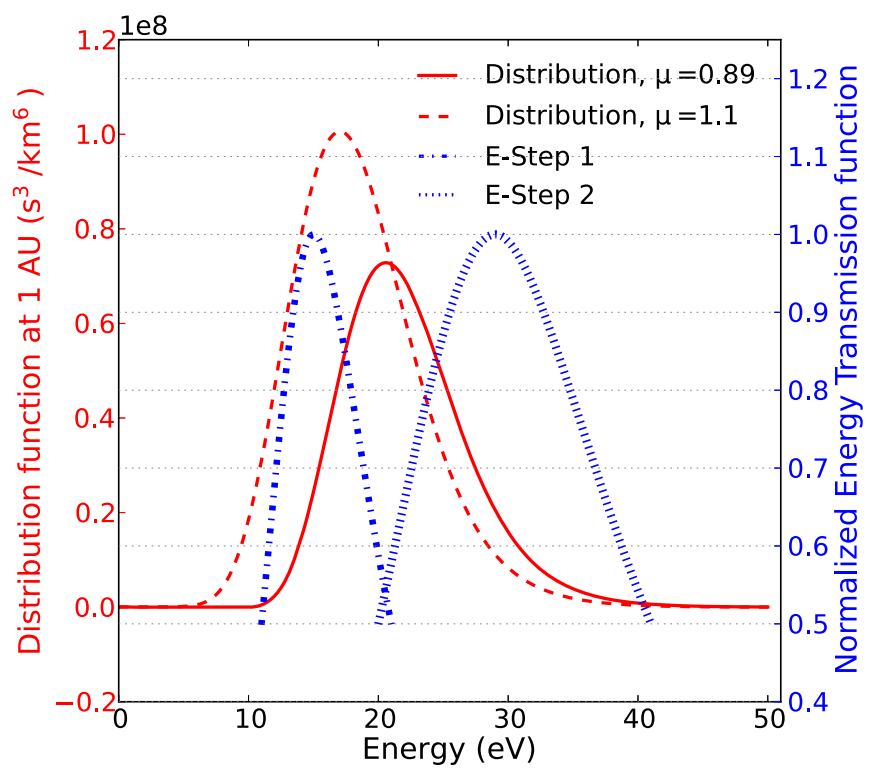

Figure 2. The distribution function of ISN H flow in the IBEX inertial frame at 1 au (for orbit 23 in 2009) is shown by the red solid line for $\mu=0.89$ and red dashed line for $\mu=1.1$. The $x$-axis shows the energy measured in the IBEX frame based on the relative velocity of incoming atoms. The normalized energy transmission function for e-step 1 is shown by the blue dashed-dotted line and for e-step 2 by the blue dotted line. As can be seen here, as $\mu$ increases, the distribution function moves toward lower energies and the e-step 1/e-step 2 ratio increases.

that the basic findings of Saul et al. $(2012,2013)$ and Schwadron et al. (2013) regarding the ratio of ISN H measured in e-steps 2 and 1 do not depend on the retrieval method.

Using a statistical analysis, Galli et al. (2019) also showed that the differences between H3-inflight- and H3-lab-retrieved ISN H are negligible for e-step 1 . The differences between the $\mathrm{H} 2 \mathrm{O} 2-$ inflight retrievals and the first two approaches are larger but still fall within the uncertainties. Using this analysis, they demonstrated the coherence of their three approaches for e-step 1. In this paper, our focus is on the temporal shift in the ISN H distribution. Therefore, we used H3-inflight retrieved data for e-step 1 ISN $\mathrm{H}$ fluxes, as it provides credible fluxes for almost an entire solar cycle.

\section{Shift of the ISN H Peak Longitude Over Time}

In this work, we used the ISN $\mathrm{H}$ data released by Galli et al. (2019; details in Section 5) at e-step 1, with one data point per orbit resolution, to find the ecliptic longitude at which the peak of the ISN $\mathrm{H}$ signal was observed $\left(\lambda_{\text {peak }}\right)$. This $\lambda_{\text {peak }}$ is calculated starting from the fall equinox, as shown in Figure 1, where $\lambda$ represents the ecliptic longitude of IBEX at the time of observation. To find $\lambda_{\text {peak }}$, we fitted a Gaussian to the observations for each year using a $\chi^{2}$ technique. In this study, we focused on spin sectors 14 and 15 , corresponding to $84^{\circ}$ and $90^{\circ}$ (the center of the spin bins from the north ecliptic pole (NEP)) $\pm 3^{\circ}$, which show the peak of the distribution in latitude, to take advantage of the higher counts in these two 


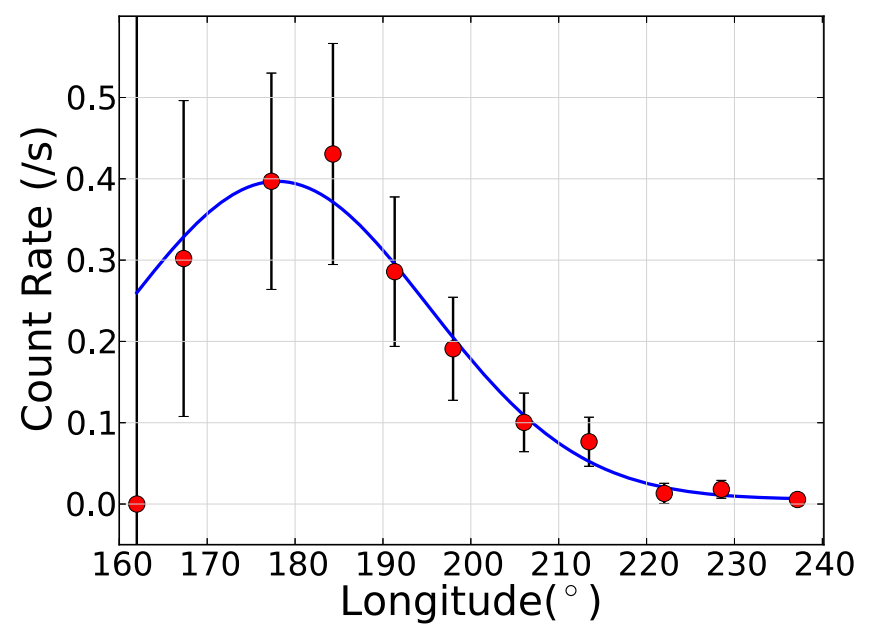

Figure 3. Gaussian function fitted to ISN H observed count rates in IBEX-Lo e-step 1 (spin sector 14) using a $\chi^{2}$ scheme for 2011.

spin sectors. Since the count rates reported for e-step 1 are more distinguishable, typically 1 order of magnitude larger than e-step 2, we concentrated on e-step 1. Figure 3 shows an example of this analysis for the ISN H season in 2011.

We used our model (aFINM) to find the parameter $\mu$ for which both the model-predicted and observed ISN H signal peak at the same longitude. To this end, we applied the same $\chi^{2}$ technique to fit a Gaussian to model predictions and find the peak longitude. We run this analysis with varying $\mu$ in iterations to reach the same peak longitude from observations for each season, obtaining an effective $\mu$ for that season. Figure 4 , as an example, compares the signal observed with $I B E X$ to our model predictions for the 2011 ISN H season. The green line in this figure represents the ISN $\mathrm{H}$ count rates predicted for the IBEXLo observation using aFINM for the actual observation times provided by Galli et al. (2019). The Gaussian, however, was fit to the black circles, which are averaged over the observation times for each orbit, to be compared with one data point per orbit data (red circles in this figure).

The orange line in Figure 4 represents aFINM-predicted count rates in the Earth reference frame with the spin axis of the instrument Sun-pointing at all times. For the same value of $\mu$, the predicted count rates, considering the actual observing conditions (green line), are different from those with continuous Sunpointing. Where no model predictions are shown in this figure over the course of an orbit, data are not available. The drift of the $I B E X$-Lo spin axis from the Sun-pointing direction over the course of an orbit leads to the sawtooth patterns in the higherresolution aFINM predictions (green line). This aspect of the observed count rates, when combined with the relative velocity of the instrument with respect to Earth and differently distributed observation times for each orbit, causes the green and orange lines in Figure 4 to deviate significantly. Particularly, for orbit 118 (fourth orbit from left), when the Sun-pointing maneuver was performed much before the data acquisition time, a significant deviation from the Gaussian fit is obvious. To avoid possible bias due to these effects, we included them in our model, and we have employed the same techniques to find the peak for both the observed signal and model predictions.

We repeated the same analysis for each year from 2009 to 2018, excluding 2015 and 2016 (see first two panels of Figure 5). In 2015, the solar activity is at its peak, resulting in the weakest ISN H signal with no statistically significant count

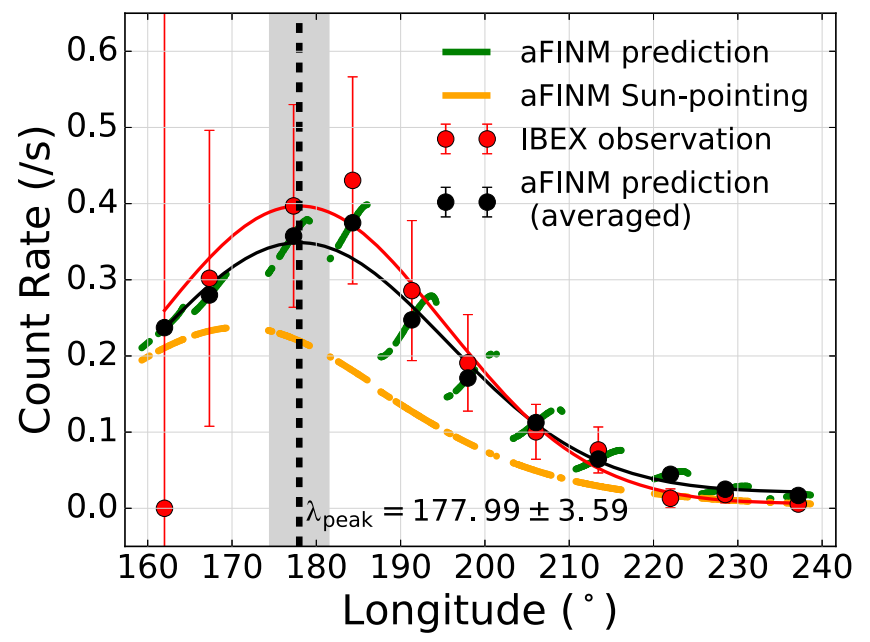

Figure 4. Model predictions based on aFINM for ISN H in 2011 are compared to observations (e-step 1, spin sector 14). The green line shows the ISN H count rate model predictions for the actual observation times provided by Galli et al. (2019). The orange line shows the ISN H count rate model predictions with the same assumptions and boundary values in the Earth reference frame, assuming the spin axis remains Sun-pointing at all times. The black line is a Gaussian fit to the black circles, which are aFINM predictions, averaged over the observation times. The red line is a Gaussian fit to the red circles, which represent ISN $\mathrm{H}$ count rates observed by IBEX. The dashed black line shows the peak longitude of the ISN H signal, with its uncertainty region in gray.

rates. Due to a different energy-stepping scheme of IBEX-Lo, the ISN H could not be extracted in the way described for 2016 (see Galli et al. 2019, for more detailed explanations). Since there are too few data points for 2013 and 2014 with values above the background (Galli et al. 2017) to allow a $\chi^{2}$ analysis, we simply used the one highest data point as the peak of the signal for those $2 \mathrm{yr}$. The crosses with huge error bars in the first two panels of Figure 5 are used to differentiate between those $2 \mathrm{yr}$ and the rest of the years for which a $\chi^{2}$ analysis is performed.

In spite of substantial uncertainties and several years for which we have no or unreliable data, a possible correlation is evident in Figure 5. The peak longitude (top panel; blue circles and orange triangles with error bars) and, in turn, the predicted parameter $\mu$ (middle panel; red circles and green triangles with error bars) appear to increase for 2009-2012. The increase persists in 2013-2014 in accordance with the solar activity. Although the high radiation pressure and ionization rates lower the ISN H signal almost down to the background level, the very faint signal seems to have shifted toward larger peak longitudes for these 2 yr. In 2017, after 2 yr of absence of operational data due to high radiation pressure and ionization rates in 2015 and a different energy-stepping scheme of IBEX-Lo in 2016, the signal returns and shows a decreasing peak longitude in two consecutive seasons, in accordance with the declining solar activity. In the middle panel of Figure 5, the $\mu_{0}=\mu\left(v_{r}=0\right)$ parameter from Kowalska-Leszczynska et al. (2018b; black line) demonstrates the same trend, although with more pronounced variations between different phases of solar activity. To better illustrate the effect of the Ly $\alpha$ radiation on the observed shift in the peak longitude, the total irradiance is also shown in the bottom panel of Figure 5.

\section{Discussion}

Early records of direct ISN $\mathrm{H}$ measurements showed an increase in the peak longitude from 2009 to 2012 as the solar 


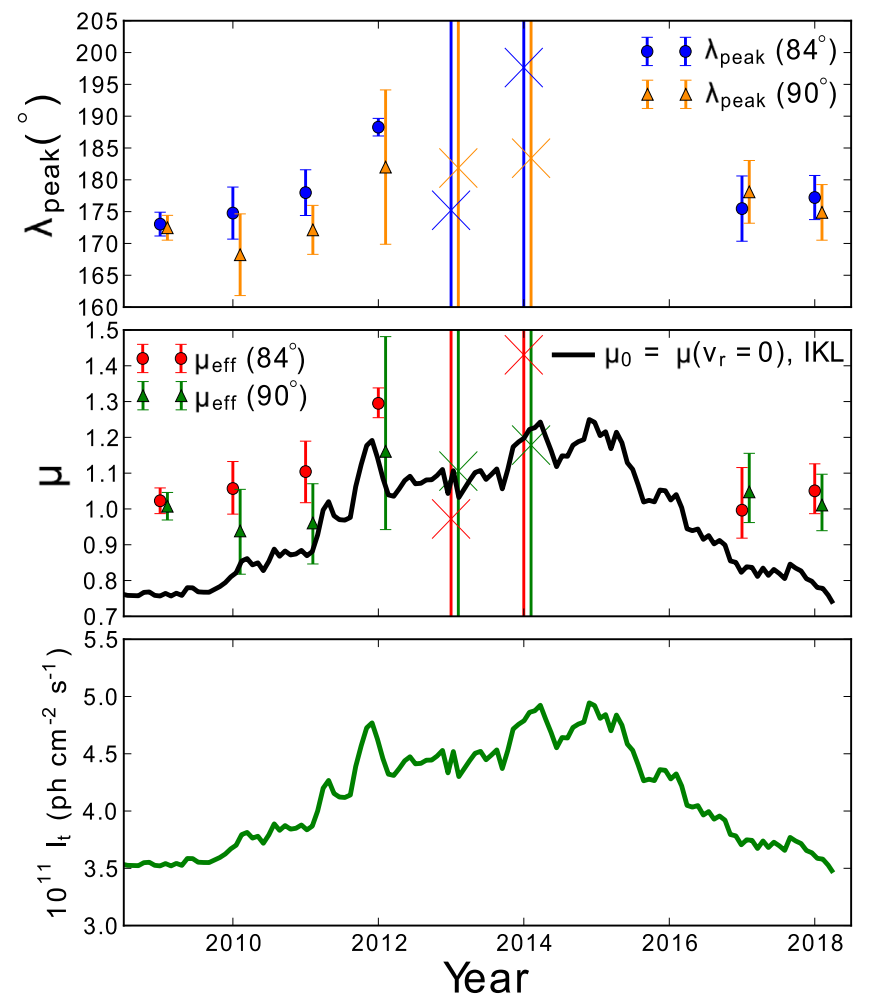

Figure 5. Top panel: ISN H flow peak longitude obtained by fitting Gaussian functions to observed ISN H count rates. Blue circles (orange triangles) with error bars show the ISN $\mathrm{H}$ flow peak longitude for spin sector 14 (15) corresponding to a $6^{\circ}$ bin with the center of the bin at $84^{\circ}\left(90^{\circ}\right)$ from the NEP. We have slightly shifted the spin sector 15 data in time to make them more visible. Crosses belong to the 2013 and 2014 seasons, for which very few data points above the background are available. We excluded these years from our $\chi^{2}$ analysis and used the highest data point as the peak of the signal. Middle panel: parameter $\mu$ that yields the best agreement between ISN $\mathrm{H}$ peak longitude from observation (top panel) and aFINM predictions in red circles (green triangles) with error bars for spin sector 14 (15), compared with $\mu_{0}$ (corresponding to the center of the profile) from the model (IKL) by KowalskaLeszczynska et al. (2018b), which is based on total irradiance (black line). Bottom panel: total irradiance from the composite solar Ly $\alpha$ flux data from LASP, integrated between 121 and $122 \mathrm{~nm}$. Since the radial velocity of ISN H atoms close to 1 au drops to values close to zero, $\mu_{0}$ can be used as a proxy for the effective radiation pressure that these atoms experience.

activity was increasing (Saul et al. 2013). They fitted a Gaussian to accumulated counts per $3 \mathrm{hr}$ in e-step 1 to find the longitude of the peak flux for each year. Based on the physical model, we expect this increase, since an increase in $\mu$ translates to either a weaker net attractive force $(\mu<1)$, which means that the atoms are bent toward the Sun at larger $\lambda_{\text {peak }}$, or a repulsive force $(\mu>1)$ that deflects the neutral atoms much earlier in their orbits (even larger $\lambda_{\text {peak }}$ ).

Despite expecting a shift in $\lambda_{\text {peak }}$ with varying solar activity, a significant trend was not observed from 2009 to 2012 ISN H data by Galli et al. (2019), using $\mathrm{H} 2 \mathrm{O} 2$ retrieved data. The lack of identifying the expected trends in the ISN $\mathrm{H}$ data can be attributed to high ISN He intensity, low ISN H intensity (particularly during years of high solar activity), and limited spatial resolution. In our analysis, we used H3-inflight retrieved data for e-step 1, which covers almost an entire solar cycle and shows a more significant longitudinal shift for the ISN H peak. We used these data because our focus is on the evolution of the spatial distribution with solar activity. The H3-inflight data spanning almost a complete solar cycle enable us to investigate the longitudinal shift of the ISN H peak through varying phases of solar activity.

The parameter $\mu$ that we obtain in this analysis is based on finding the same peak longitude with IBEX-Lo observations as explained in Section 6. In this way, we do not need to incorporate any physical model for the parameter $\mu$ and its dependence on time, radial speed, or heliolatitude. In this analysis, $\mu$ is an input parameter in our model, which is stationary, since our model only permits constant values. As found in this way, $\mu$ may be taken as an effective $\mu$ for each observation season.

However, ISN $\mathrm{H}$ atoms are on their journey to the inner heliosphere for about $20 \mathrm{yr}$ and experience almost two full solar cycles. As a result, variations of the radiation pressure with time and radial speed can play an essential role during the journey of $\mathrm{H}$ atoms from outside the heliosphere to $1 \mathrm{au}$, challenging stationary models. Tarnopolski \& Bzowski (2009) compared the WTPM model predictions for ISN $\mathrm{H}$ density between a flat radiation profile (i.e., no radial speed dependence of $\mu$ ) and a nonflat profile for equivalent Ly $\alpha$ intensities and showed that the differences are quite substantial at 1 au. More recently, Kowalska-Leszczynska et al. (2018a) applied a newer model of the radiation profile (Kowalska-Leszczynska et al. $2018 b$ ) and showed that the differences between the predictions of these two models are significant, concluding that the ISN $\mathrm{H}$ signal observed by $I B E X$ is sensitive to radiation pressure variations with radial velocity.

This raises doubts if we need to account for an integral of the radiation pressure affecting the trajectory. However, KowalskaLeszczynska et al. (2018a) showed that at $\sim 20$ au from the Sun, the environment becomes optically thick, and thus variations of radiation pressure are of secondary importance beyond this region. In the Appendix, we justify the use of a stationary model, showing that the net force acting on ISN $\mathrm{H}$ atoms is negligible until the last few months of their travel so that temporal variations of $\mu$ can be neglected. The absence of a time lag between the solar activity phase and variations in ISN $\mathrm{H}$ intensity reported by Galli et al. (2019) also confirms that the effect of radiation pressure and loss processes becomes most important close to the Sun, and an integration of these effects over the entire trajectory is not necessary. ${ }^{8}$

The qualitative agreement between $\mu_{0}$ from KowalskaLeszczynska et al. (2018b) and the effective $\mu$ obtained in this analysis (middle panel of Figure 5) may indicate that these two parameters are tightly connected. To investigate this further, we illustrate $\mu_{\text {eff }}$ (from this study) along with $\mu_{0}, \mu\left(v_{r}=-\right.$ $25 \mathrm{~km} \mathrm{~s}^{-1}$; minus sign means toward the Sun), and $\mu\left(v_{r}=-35 \mathrm{~km} \mathrm{~s}^{-1}\right)$ based on the Ly $\alpha$ profile (KowalskaLeszczynska et al. 2018b) in Figure 6. As can be seen in this figure, the best agreement is found between $\mu_{\mathrm{eff}}$ and $\mu\left(v_{r}=-35 \mathrm{~km} \mathrm{~s}^{-1}\right)$. However, it is very unlikely that the majority of ISN $\mathrm{H}$ atoms have an average radial velocity close to $-35 \mathrm{~km} \mathrm{~s}^{-1}$ in the last phases of their journey. In particular, as detailed in the Appendix, at small helioradii, where the radiation pressure becomes important, the radial velocity decreases dramatically. For an ISN $\mathrm{H}$ atom, starting at $100 \mathrm{au}$ with ISN bulk velocity, the weighted average of $v_{r}$ in the last 6 months of the travel is about $-18.3 \mathrm{~km} \mathrm{~s}^{-1}$. Since this value

\footnotetext{
8 The time lag between radiation pressure variations and resulting variations in the ISN $H$ helioglow at $\sim 1$ au was shown to exist by Bzowski et al. (2002). However, they showed that it was at its minimum value in the upwind direction.
} 


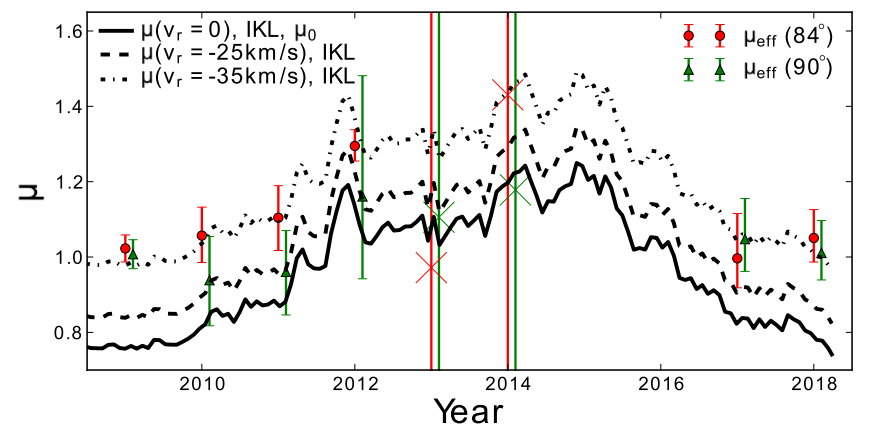

Figure 6. The $\left(\mu_{\text {eff }}\right)$ obtained from our analysis (red circles with error bars for spin sector 14 and green triangles with error bars for spin sector 15) is compared with $\mu_{0}, \mu\left(v_{r}=-25 \mathrm{~km} \mathrm{~s}^{-1}\right)$, and $\mu\left(v_{r}=-35 \mathrm{~km} \mathrm{~s}^{-1}\right)$ from Kowalska-Leszczynska et al. (IKL; 2018b). While we expect a quantitative agreement between $\mu_{\mathrm{eff}}$ and $\mu_{0}$ from IKL (see the Appendix), $\mu_{\mathrm{eff}}$ is closest to $\mu$ $\left(v_{r}=-35 \mathrm{~km} \mathrm{~s}^{-1}\right)$. As in Figure 5, we have slightly shifted the spin sector 15 data to make them more visible.

falls into the flat part of the radiation profile, as explained further in the Appendix, $\mu_{0}$ is a good approximation for the effective $\mu$ acting on the $\mathrm{H}$ atoms. In conclusion, the difference between the $\mu_{\text {eff }}$ obtained in this analysis and any $\mu$ from Ly $\alpha$ based models is inherent and most likely associated with absolute calibration of Ly $\alpha$ line profiles.

There is an evident linear correlation between the total Ly $\alpha$ irradiance and the central irradiance of the solar Ly $\alpha$ profile, and $\mu_{0}$ as a result (see Figure 7 from Lemaire et al. 2015). To further investigate the effective parameter $\mu$ from our analysis, we illustrate its correlation with the total Ly $\alpha$ irradiance in Figure 7. The $\mu_{0}$ in this figure was calculated based on the total $\operatorname{Ly} \alpha$ irradiance averaged over the two Carrington cycles right before the ISN $\mathrm{H}$ season in that year, to provide the best estimate for the effective radiation pressure that ISN $\mathrm{H}$ atoms experience (see the Appendix). The linear fit (purple line; $\left.\mu_{\text {eff }}=(0.27 \pm 0.03) I_{t}+(0.03 \pm 0.10)\right)$ to $\mu_{\text {eff }}$ (blue circles with error bars for spin sector 14 and red circles with error bars for spin sector 15) is compared with $\mu_{0}$ (green line; $\mu_{0}=$ $\left.(0.35 \pm 0.001) I_{t}-(0.46 \pm 0.005)\right)$.

The green shaded area in Figure 7 indicates the uncertainty region for $\mu_{0}$ (green line) based on a $15 \%$ uncertainty estimated for observed Ly $\alpha$ line profiles (Lemaire et al. 2015; KowalskaLeszczynska et al. 2018b). The purple shaded area demonstrates the uncertainty region associated with a fit to our predicted $\mu_{\text {eff }}$ (purple line). The two shaded uncertainty regions overlap, indicating an agreement between $\mu_{\text {eff }}$ based on ISN H data observed by IBEX and $\mu_{0}$ from Ly $\alpha$ data, despite the steeper slope for $\mu_{0}$. This might suggest that during years of low solar activity, when radiation pressure is less effective in decelerating ISN $\mathrm{H}$ atoms, atoms from the faster wing of the ISN $\mathrm{H}$ distribution are not decelerated enough to fall in the flat part of the Ly $\alpha$ line profile so that $\mu_{\text {eff }}$ deviates from $\mu_{0}$. Another possible explanation is a systematic oversubtraction of ISN He in ISN H retrieval methods (Section 5), which shifts the peak longitude toward later orbits. This oversubtraction would affect the ISN H flux more dramatically during years of solar minimum, since there is a wider overlap between ISN $\mathrm{H}$ and $\mathrm{He}$ signals in those years. The black dashed line in Figure 7 is obtained by fitting a line to $\mu_{\text {eff }}$ assuming the same slope as for $\mu_{0}$ to find the intercept $(-0.24 \pm 0.01)$. The $0.22 \pm 0.014$ difference between the green line and dashed black line intercepts could be indicative of absolute calibration issues with Ly $\alpha$ line profiles.

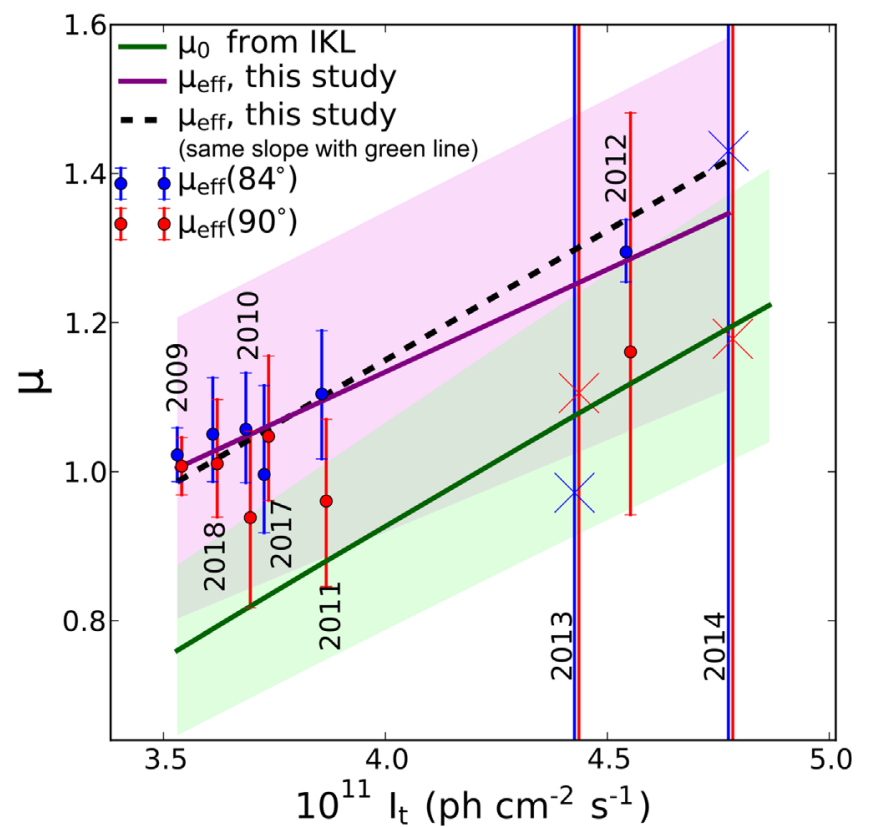

Figure 7. The ( $\left.\mu_{\text {eff }}\right)$ obtained from our analysis (blue circles with error bars for spin sector 14 and red circles with error bars for spin sector 15) vs. total irradiance from LASP data, integrated between 121 and $122 \mathrm{~nm}$ and averaged over two Carrington cycles before the ISN H season for each year. As in previous figures, we have slightly shifted the spin sector 15 data to make them more visible. The linear relationship between $\mu_{0}$ from Kowalska-Leszczynska et al. (2018b; $\mu_{0}$ from IKL) and total irradiance is shown by the green line with an estimated $15 \%$ uncertainty (green shaded area). The purple line shows a linear fit to all $\mu_{\text {eff }}$ points from our analysis for sectors 14 and 15 using a $\chi^{2}$ scheme, with the purple shaded region indicating the uncertainty. In addition to the evident nonzero slope of this line, which demonstrates a correlation between $\mu_{\text {eff }}$ and total irradiance and thus adds credibility to our analysis, the overlap of the uncertainty regions presents a quantitative agreement never reported previously based on $I B E X$ data. However, the smaller slope of the purple line in comparison with the green line indicates that variations with total irradiance are less noticeable in $\mu_{\text {eff }}$ than in $\mu_{0}$ from IKL. The black dashed line shows a linear fit to the $\mu_{\text {eff }}$ points using the same slope as $\mu_{0}$ (green line) to find the intercept using a $\chi^{2}$ scheme. The $0.22 \pm 0.014$ difference between the intercepts of these two lines can be associated with the absolute calibration issue of $\operatorname{Ly} \alpha$ line profiles.

Katushkina et al. (2015) found a qualitative difference between their predictions and IBEX data. They found that, contrary to the $I B E X$ data, their model results obtain higher rates for e-step 2 than e-step 1 in the expected range of radiation pressure and ionization rate. The same discrepancy has been reported for all existing models described in Section 3 in the expected range of radiation pressure and ionization rate (e.g., see Katushkina et al. 2015; Galli et al. 2019). Such a discrepancy limits the analysis of the intensity of the ISN $\mathrm{H}$ signal, although it does not affect the longitudinal shift of its peak. This discrepancy could be associated with a poorly known energy response function, uncertainties in the total irradiance data, oversubtraction of ISN He in e-step 2 in data retrieval methods from previous work, or some physical aspects being neglected in our current models.

A $\chi^{2}$ analysis to obtain the best fit between model-predicted ratio of fluxes in the two energy channels with IBEX data showed that the most important parameter affecting this ratio is the radiation pressure (Katushkina et al. 2015). Also, as can be seen in Figure 2, since the distribution peak of the ISN $\mathrm{H}$ at 1 au falls between the two lowest e-steps, its signal is very sensitive to solar cycle variations. More specifically, variations in $\mu$ move the distribution function observed at 1 au in energy 
and thus change the flux ratio between e-steps 1 and 2 . This suggests that this qualitative difference between model predictions and IBEX data could be related to the absolute calibration of the radiation pressure, at least in part.

Another explanation for the remaining discrepancy might be a retrieval bias against ISN $\mathrm{H}$ in e-step 2 or an unexpected behavior of the energy response function in flight compared to laboratory calibration. As detailed in Section 5, Galli et al. (2019) developed three different retrieval methods to derive ISN $\mathrm{H}$ intensity more accurately. Their obtained ratio between the two e-steps confirms previous reports (Saul et al. 2013; Schwadron et al. 2013) and thus excludes oversubtraction of the ISN $\mathrm{He}$ in e-step 2 as a possible explanation for the aforementioned discrepancy. However, a systematic oversubtraction of ISN He in both e-steps might still have caused the less noticeable variations of $\mu_{\mathrm{eff}}$ in comparison with $\mu_{0}$ (smaller slope of purple line compared to green line in Figure 7) by eliminating the actual peak for the years of low solar activity. This can be a plausible explanation, since an oversubtraction of ISN $\mathrm{He}$ is expected to affect e-step 2 more substantially than e-step 1, leading to the previously mentioned discrepancy.

The poor knowledge of the IBEX-Lo response function for ISN $\mathrm{H}$ is likely due to different behavior of IBEX-Lo in space than in laboratory calibrations at energies below $50 \mathrm{eV}$. At such low energies, surface science effects play a significant role in the scattering and ionization of particles. The conversion surface might have become chemically contaminated during launch, and this contamination has become permanent because it was burned in by UV light early in the mission (Riedo et al. 2010) and/or because of chemical reactions with the surface (hydrazine). This has possibly led to a different surface coating on IBEX-Lo, which is stable over the years but deviates from laboratory conditions. In the future, we plan to perform a comprehensive investigation of the energy channels, their associated response functions to the ISN $\mathrm{H}$ signal, and how improving them affects the ISN $\mathrm{H}$ count rates, including information from the in-flight performance in response to ISN H. In our future work, we will also focus more on the latitudinal profile of the ISN H signal, as the narrower ISN He distribution cannot contaminate it (Galli et al. 2019).

Since the beginning of the IBEX mission, comparing models (briefly discussed in Section 3) against IBEX-Lo observed fluxes for the ISN $\mathrm{H}, \mathrm{He}$, and $\mathrm{O}$ was used to find the essential parameters associated with the ISN flow, most importantly for the ISN He flow, which is least affected by ionization losses due to its high ionization potential. For $\mathrm{H}$ atoms, the radiation pressure was also investigated in this way. Fitting aFINM to 2009-2011 data obtained a slightly increasing $\mu$ from $0.94 \pm 0.04$ in 2009 to $1.01 \pm 0.05$ in 2011 (Schwadron et al. 2013). A combination of the global heliospheric interface model and the ISN $\mathrm{H}$ distribution inside the heliosphere, limited to orbit 23 of IBEX (2009), obtained $\mu=1.26_{-0.076}^{+0.06}$ (Katushkina et al. 2015). Although their obtained parameter was much larger than $\mu_{0}=0.89$, derived from the integrated solar Ly $\alpha$ irradiance, the first report of direct detections of ISN $\mathrm{H}$ had already predicted a parameter $\mu$ larger than unity for 2009-2010 (Saul et al. 2012).

Investigating variations in the ISN $\mathrm{H}$ intensities and their longitudinal shift throughout different phases of the solar cycle can certainly be beneficial in resolving the absolute calibration issue of the radiation pressure. In the present study, we focused on the longitudinal shift of the ISN $\mathrm{H}$ signal due to the aforementioned discrepancy between all existing models and the signal observed by $I B E X$. We created a methodology to find yearly estimates of effective radiation pressure. We found the effective parameter $\mu$ increasing from values as low as $0.94_{-0.12}^{+0.12}$ (for spin sector 15 in 2010) during the cycle 23 minimum to values as high as $1.29_{-0.04}^{+0.04}$ (for spin sector 14 in 2012) during the cycle 24 maximum and then decreasing again to $0.996_{-0.040}^{+0.043}$ (for spin sector 14 in 2017), averaged at $1.074 \pm 0.038$ through the full solar cycle. These results based on IBEX data demonstrate, for the first time, a qualitative agreement (as well as a quantitative agreement, within the uncertainties) with simulations of the Ly $\alpha$ profile based on the total irradiance observations. However, the parameter $\mu$ from our analysis averaged over cycle 24 is $\sim 21 \%$ larger than the average of $\mu_{0}$ from Kowalska-Leszczynska et al. (2018b) over the same time interval. The continuation of this type of analysis, along with the ISN $\mathrm{H}$ observations with IBEX-Lo, particularly as we are heading toward a solar minimum in the next few years, will play an essential role in understanding the ISN $\mathrm{H}$ flow distribution function, as well as the radiation pressure and ionization processes to which it is subjected.

\section{Summary and Conclusion}

While the ISN flow has been studied extensively through sophisticated modeling and comparing results with IBEX data, most of these studies have focused on ISN He. The radiation pressure, with its temporal and spatial variations and more effective ionization processes, complicates the analysis of ISN H flow. So far, this problem has been addressed in a limited number of studies (Schwadron et al. 2013; Katushkina et al. 2015).

A new release of ISN $\mathrm{H}$ comprehensive maps observed by IBEX (Galli et al. 2019), which showed the reappearance of the signal after the solar maximum of cycle 24 , motivated us to revise aFINM and compare its results with IBEX observations throughout the past $10 \mathrm{yr}$. Although the ongoing discrepancy in the ratio of the ISN H counts in the lowest two energy channels between all available models and the observations makes it difficult to gain information from the signal intensity, the longitudinal shift is still a reasonable indicator of the ISN $\mathrm{H}$ signal variations with solar activity.

Based on our current physical understanding of the ISN $\mathrm{H}$ flow, we expect a shift in the peak longitude that increases with solar activity. The expected shift, however, currently still carries large uncertainties. This could be most likely due to very low intensities of ISN H; large uncertainties, especially during years of high solar activity; poorly known response functions of the instrument for such low energy; oversubtraction of ISN He in e-steps 1 and 2; or some of the physics of the problem being neglected in our current models. In spite of the large uncertainties, not only is the anticipated shift observed, the effect also suggests that the radiation pressure generally exerts a larger force than gravity. The trend that we observe here and the fact that it agrees qualitatively with total irradiance intensities and the parameter $\mu$ obtained from an independent model indicate that a better-known response function of the instrument or higher-resolution data could lead us to observe the predicted shift and even more confidently address the calibration issue with the absolute irradiance of solar Ly $\alpha$ fluxes. Further study of the IBEX data and the instrument response to $\mathrm{H}$ atoms will help to refine our understanding of solar radiation pressure and its effect on ISN $\mathrm{H}$ distributions.

The current anomalously low solar activity indicates that we may be entering a period of persistent decline in the 
heliospheric magnetic field (Rahmanifard et al. 2017). Selected for launch in 2024, the Interstellar Mapping and Acceleration Probe (IMAP) will provide an opportunity to track the interaction between the heliosphere and the VLISM through these years of unprecedentedly low solar activity (McComas et al. 2018). IMAP will address our ongoing issues with detecting the ISN $\mathrm{H}$ signal and its essential features, including the expected peak longitude shift, through improving time resolution, increasing angular coverage and sensitivity, and better suppressing the background (Schwadron et al. 2016b).

We thank all of the outstanding men and women who have made the IBEX mission such a wonderful success. This work was carried out as a part of the $I B E X$ project, with support from NASA's Explorer Program. A.G. and P.W. thank the Swiss National Science Foundation for financial support. E.M. is grateful for support from ISSI for an extended visit in Bern during the formative stage of this study. Research at SwRI was funded by subcontract from Princeton University. M.B., M.A.K., I.K.-L., and J.M.S. were supported by Polish NCN grant 2018/M/ST9/00036. F.R. acknowledges useful discussions with $\mathrm{O}$. A. Katushkina in the early stages of this project.

\section{Appendix \\ Justification for Using a Stationary Model for the ISN H Problem}

The radiation pressure is an outward force in the radial direction exerted on the ISN $\mathrm{H}$ atoms due to resonant absorption and reemission of Ly $\alpha$. It roughly compensates for solar gravity, which prevents significant gravitational focusing of ISN $\mathrm{H}$ atoms. Therefore, a longitudinal shift of ISN $\mathrm{H}$ is reported with respect to ISN He. Moreover, the temporal variations of the radiation pressure at different stages of solar activity are expected to cause a signal shift for each season. The parameter $\left(\mu=\left|F_{\text {rad }}\right| /\left|F_{g}\right|\right)$ is a function of time $(t)$, radial speed $\left(v_{r}\right)$, and heliolatitude $(\theta)$. The shape of $\mu$ with respect to $v_{r}$ projects the shape of the Ly $\alpha$ profile.

Previous estimations of $\mu$ based on ISN H IBEX observations yielded unexpectedly high values (Saul et al. 2013; Katushkina et al. 2015). To address these high values, Kowalska-Leszczynska et al. (2018b) modeled the solar Ly $\alpha$ profile based on observations from SUMER/SOHO (Lemaire et al. 2015). Their model predicts three functions featuring different elements of the profile. Their model consists of a kappa function featuring the main emission line produced in the chromosphere, a negative Gaussian function featuring the absorption in the transition region, and a linear function featuring the spectral background. Figure 8 shows the average radiation profile over one Carrington rotation (using total irradiance, integrated between 121 and $122 \mathrm{~nm}$, from LASP) during the ISN H observation seasons in 2009, 2012, and 2017. The profiles in this figure clearly show the distinction between the $\mu$ values at different phases of the solar activity.

The effects of using such a model on the ISN H IBEX observations and their interpretation were studied in a more recent paper (Kowalska-Leszczynska et al. 2018a). The integrated irradiance used in Kowalska-Leszczynska et al. (2018b) is based on observations from SUMER/SOHO with daily resolution. However, proxies are used to fill the occasional gaps. The UARS/SOLSTICE (Woods et al. 1996, 2000) data are used for the absolute calibration, and observations from other sources are scaled to this calibration.

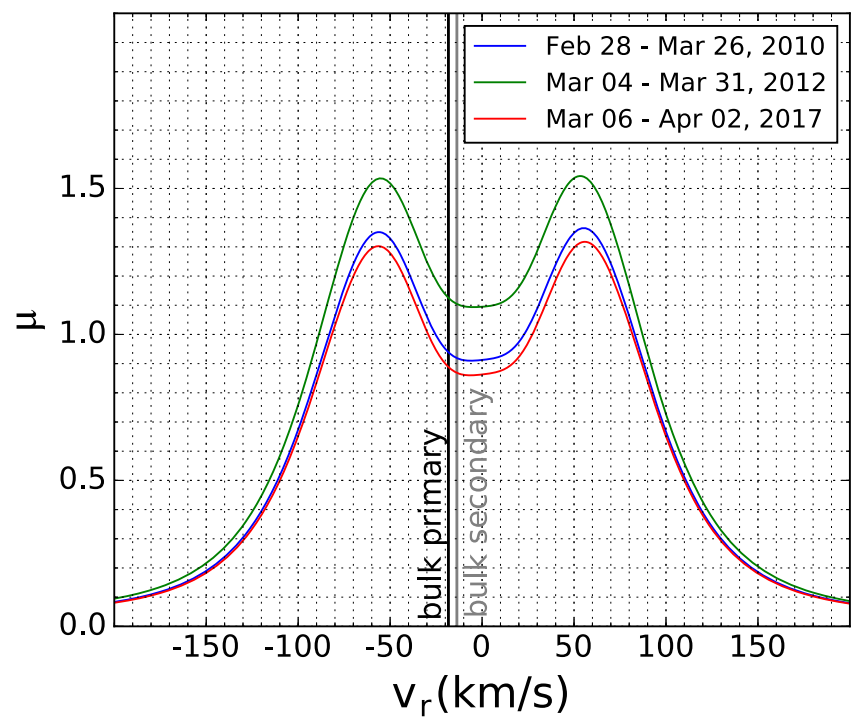

Figure 8. The Ly $\alpha$ radiation profile as a function of $v_{r}$ based on the KowalskaLeszczynska et al. (2018b) model obtained for the total irradiance averaged over one Carrington cycle for 2009, 2012, and 2017. The difference between solar minimum and solar maximum is significant. The black (gray) vertical line shows the averaged radial velocity of the bulk primary (secondary) atoms in the last 6 months of their journey for ISN $\mathrm{H}$ atoms that reach 1 au during the solar minimum condition.

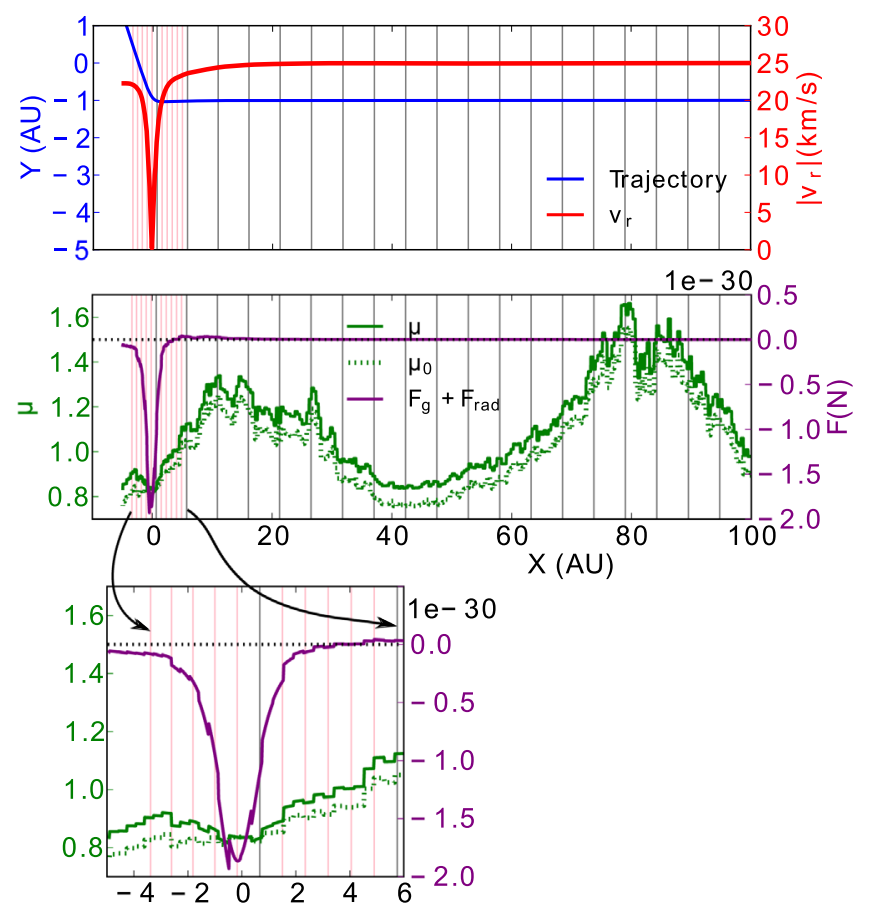

Figure 9. Simulated trajectory, radial velocity (top panel), net force, $\mu$, and $\mu_{0}$ from the Kowalska-Leszczynska et al. (2018b) model (middle panel) for a hypothetical $\mathrm{H}$ atom that starts from $x=100 \mathrm{au}$ and $y=-1$ au with $v_{x}=-25 \mathrm{~km} \mathrm{~s}^{-1}$ and $v_{y}=0$ in 1998. The temporal variation of $\mu$ does not significantly affect the negligible net force far from the Sun. Therefore, the radial velocity remains constant, and the $\mathrm{H}$ atom travels on a straight line until it gets close to the Sun, where the net force becomes large enough to reflect the temporal variations of $\mu$ while the radial velocity drops to zero almost abruptly. As can be seen here, this happens during the last 2 months of the trajectory (gray vertical lines represent years, and pink vertical lines separate 2 month periods) before the $\mathrm{H}$ atom reaches $I B E X$. More interestingly, during this phase of the journey, $\mu$ almost equals $\mu_{0}$, indicating that $\mu_{0}$ is a good approximation for $\mu$ and the ISN $\mathrm{H}$ trajectory can be approximated by a hyperbola (bottom panel). Because solar minimum conditions are reached at this point, the ISN H atom is deflected toward the Sun. 


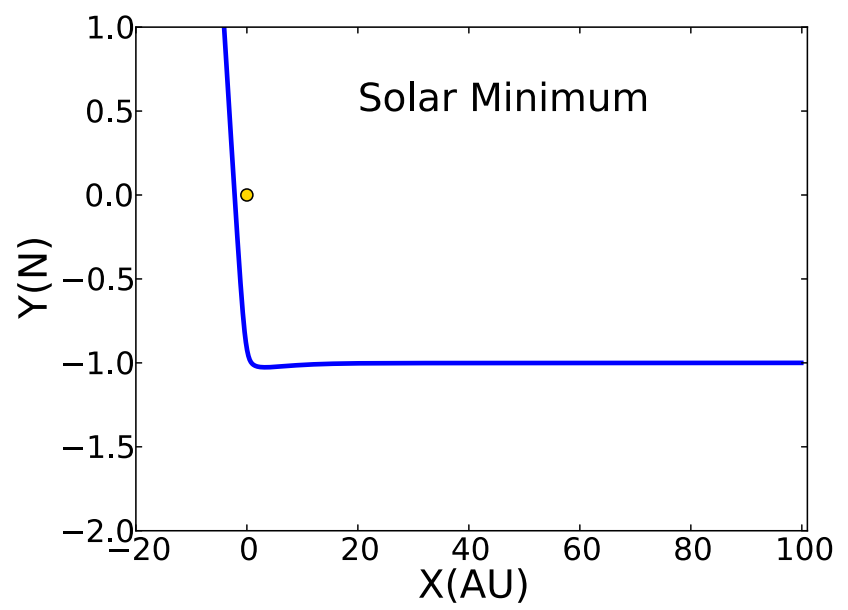

(a)

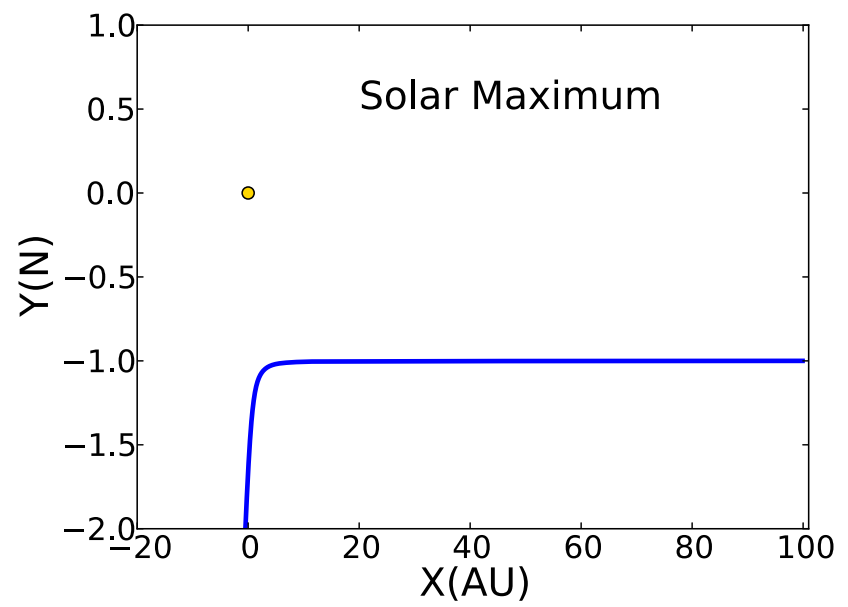

(b)

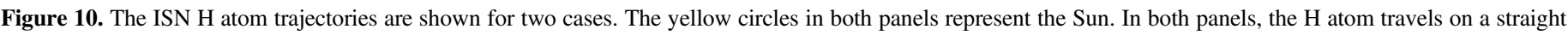

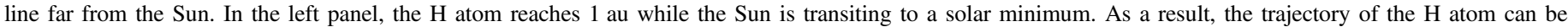

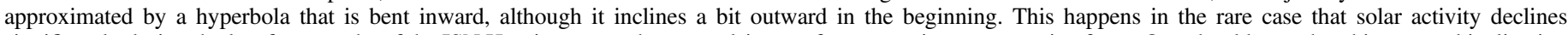

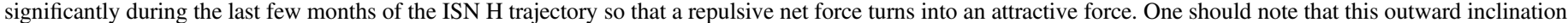

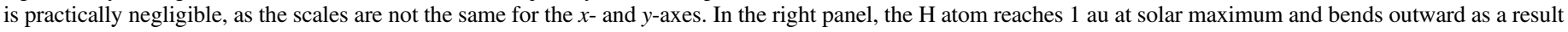
of the repulsive net force.

An estimated $\sim 15 \%$ uncertainty has been suggested for the total uncertainty of the observed profiles (Lemaire et al. 2015; Kowalska-Leszczynska et al. 2018b). However, the absolute calibration of the integrated Ly $\alpha$ irradiance and its effect on the predicted Ly $\alpha$ radiation profile has remained an issue.

On their journey from the edge of the heliosphere to $1 \mathrm{au}$, ISN $\mathrm{H}$ atoms take about $20 \mathrm{yr}$. They are subject to temporal variations in the radiation pressure at different phases of the solar activity. To investigate this effect on the ISN $\mathrm{H}$ trajectories and their observed peak longitude at $1 \mathrm{au}$, we developed a numerical tool that accounts for temporal and radial velocity variations of the radiation pressure based on the Kowalska-Leszczynska et al. (2018b) model. This tool solves the equation of motion for a single ISN $\mathrm{H}$ atom numerically.

We simulated the speed, trajectory, parameter $\mu$, and net force acting on an ISN $\mathrm{H}$ atom, which travels toward the inner heliosphere at a speed of $25 \mathrm{~km} \mathrm{~s}^{-1}$ at $100 \mathrm{au}$. Assuming that this particle starts in 1998 at $100 \mathrm{au}$, the parameter $\mu$ and the net force acting on the particle are shown in the middle panel of Figure 9. As can be seen, far from the Sun, the net force acting on the ISN H atoms (Equation (1)) is almost zero, so temporal variations of $\mu$ do not significantly affect the negligible net force. Therefore, ISN $\mathrm{H}$ atoms travel on a straight line until they get very close to 1 au during the last few months of their journey (top panel of Figure 9). This is because the radiation force $\left(F_{\mathrm{rad}}\right)$ is proportional to $1 / r^{2}$ as the UV photon flux.

Over the last few au of the ISN $\mathrm{H}$ atom trajectory (last 2 months; see pink vertical lines), ISN $\mathrm{H}$ atoms are finally accelerated (or decelerated) by the attractive (or repulsive) net force. At this point, the net force becomes substantial and reflects temporal variations of $\mu$. However, the radial velocity component of the particles drops to values close to zero, and $\mu$ approaches $\mu_{0}$ very closely (bottom panel of Figure 9). As a result, the flat part in the center of the radiation profile (Figure 8) is the only part of the profile that ISN $\mathrm{H}$ atoms are effectively exposed to; thus, $\mu_{0}$ is a good proxy for the effective $\mu$. Therefore, the trajectory of the atoms can be treated as a hyperbola with a constant $\mu_{0}$. This means that a stationary model using $\mu$ values appropriately averaged over the last phases of the ISN $\mathrm{H}$ journey can address the effect of the radiation pressure on their trajectory.

Once the ISN $\mathrm{H}$ atoms get close enough to the Sun, the hyperbolic characteristics of their trajectories become important, bending them inward or outward depending on the current phase of the solar activity, as shown in Figure 10. This figure is made with the numerical tool introduced in this section and thus takes the radiation pressure variations into account.

\section{ORCID iDs}

F. Rahmanifard (10) https://orcid.org/0000-0001-9316-0553

E. Möbius (iD https://orcid.org/0000-0002-2745-6978

N. A. Schwadron (i) https://orcid.org/0000-0002-3737-9283

A. Galli (iD https://orcid.org/0000-0003-2425-3793

J. M. Sokół (1) https://orcid.org/0000-0002-4173-3601

M. Bzowski (iD https://orcid.org/0000-0003-3957-2359

I. Kowalska-Leszczynska (ib https://orcid.org/0000-00026569-3800

M. A. Kubiak (iD https://orcid.org/0000-0002-5204-9645

P. Wurz (iD https://orcid.org/0000-0002-2603-1169

S. A. Fuselier (iD https://orcid.org/0000-0003-4101-7901

D. J. McComas (i) https://orcid.org/0000-0001-6160-1158

\section{References}

Axford, W. I. 1972, NASSP, 308, 609

Baranov, V. B., \& Malama, Y. G. 1993, JGR, 98, 15157

Blum, P. W., \& Fahr, H. J. 1970, A\&A, 4, 280

Bochsler, P., Kucharek, H., Möbius, E., et al. 2014, ApJS, 210, 12

Bzowski, M., Fahr, H. J., Rucinski, D., \& Scherer, H. 1997, A\&A, 326, 396

Bzowski, M., Kubiak, M. A., Hłond, M., et al. 2014, A\&A, 569, A8

Bzowski, M., Kubiak, M. A., Möbius, E., et al. 2012, ApJS, 198, 12

Bzowski, M., Mäkinen, T., Kyrölä, E., et al. 2003, A\&A, 408, 1165

Bzowski, M., Möbius, E., Tarnopolski, S., Izmodenov, V., \& Gloeckler, G. 2008, A\&A, 491, 7

Bzowski, M., \& Rucinski, D. 1995, SSRv, 72, 467

Bzowski, M., Sokół, J. M., Tokumaru, M., et al. 2013, in Cross-Calibration of Far UV Spectra of Solar System Objects and the Heliosphere, ed. R. M. Bonnet, E. Quemerais, \& M. Snow, Vol. 13 (New York: Springer), 67 Bzowski, M., Summanen, T., Ruciński, D., et al. 2002, JGR, 107, A7

Bzowski, M., Swaczyna, P., Kubiak, M. A., et al. 2015, ApJS, 220, 28 
Fahr, H. J. 1968, Ap\&SS, 2, 474

Fahr, H. J. 1971, A\&A, 14, 263

Fahr, H. J. 1979, A\&A, 77, 101

Frisch, P. C., Bzowski, M., Grun, E., et al. 2009, SSRv, 146, 235

Fuselier, S. A., Bochsler, P., Chornay, D., et al. 2009, SSRv, 146, 117

Galli, A., Wurz, P., Rahmanifard, F., et al. 2019, ApJ, 871, 52

Galli, A., Wurz, P., Schwadron, N. A., et al. 2017, ApJ, 851, 2

Gloeckler, G., \& Geiss, J. 2001, in The 3-D Heliosphere at Solar Maximum, ed. R. G. Marsden, 97 (Dordrecht: Springer), 169

Gloeckler, G., Möbius, E., Geiss, J., et al. 2004, A\&A, 426, 845

Izmodenov, V. 2001, in The Outer Heliosphere: The Next Frontiers ed. K. Scherer et al. (Amsterdam: Pergamon Press), 23

Izmodenov, V. V., \& Alexashov, D. B. 2015, ApJS, 220, 32

Izmodenov, V. V., \& Baranov, V. B. 2006, in The Physics of the Heliospheric Boundaries, ed. R. Kallenbach (Paris: ESA), 67

Izmodenov, V. V., \& Malama, Y. G. 2004, in AIP Conf. Proc. 719, Physics of the Outer Heliosphere, ed. V. Florinski, N. V. Pogorelov, \& G. P. Zank (Melville, NY: AIP), 47

Katushkina, O. A., Izmodenov, V. V., Alexashov, D. B., Schwadron, N. A., \& Mccomas, D. J. 2015, ApJS, 220, 33

Kowalska-Leszczynska, I., Bzowski, M., \& Kubiak, M. A. 2018a, ApJ, 868, 49

Kowalska-Leszczynska, I., Bzowski, M., Sokół, J. M., \& Kubiak, M. A. 2018b, ApJ, 852, 115

Kubiak, M. A., Bzowski, M., Sokół, J. M., et al. 2014, ApJS, 213, 29

Kubiak, M. A., Swaczyna, P., Bzowski, M., et al. 2016, ApJS, 223, 13

Lallement, R., \& Bertin, P. 1992, A\&A, 266, 479

Lallement, R., Quémerais, E., Bertaux, J. L., et al. 2005, Sci, 307, 1447

Lallement, R., Quémerais, E., Koutroumpa, D., et al. 2010, in ASP Conf. Ser. 428, SOHO-23: Understanding a Peculiar Solar Minimum, ed. S. R. Cranmer, J. T. Hoeksema, \& J. L. Kohl (San Francisco, CA: ASP), 253

Lallement, R., Raymond, J. C., Bertaux, J.-L., et al. 2004, A\&A, 426, 867

Lee, M. A., Kucharek, H., Eberhard, M., et al. 2012, ApJ, 198, 10

Lee, M. A., Möbius, E., \& Leonard, T. W. 2015, ApJS, 220, 23

Lemaire, P., Vial, J.-C., Curdt, W., Schühle, U., \& Wilhelm, K. 2015, A\&A, 581, A26

Lindsay, B. G., \& Stebbings, R. F. 2005, JGRA, 110, A12213

Linsky, J. L., Brown, A., Gayley, K., Diplas, A., \& S, B. D. 1993, ApJ, 402, 694

McComas, D. J., Alexashov, D., Bzowski, M., et al. 2012, Sci, 336, 1291

McComas, D. J., Allegrini, F., Bochsler, P., et al. 2009, SSRv, 146, 11

McComas, D. J., Allegrini, F., Bzowski, M., et al. 2014, ApJS, 213, 20

McComas, D. J., Bzowski, M., Fuselier, S. A., et al. 2015, ApJS, 220, 22

McComas, D. J., Christian, E. R., Schwadron, N. A., et al. 2018, SSRv, 214,116

McComas, D. J., Ebert, R. W., Elliott, H. A., et al. 2008, GeoRL, 35, L18103
McComas, D. J., Elliott, H. A., Gosling, J. T., \& Skoug, R. M. 2006, GeoRL, 33, L09102

Mccomas, D. J., Elliott, H. A., Schwadron, N. A., \& Goldstein, B. E. 2003, GeoRL, 30, 1517

Meier, R. R. 1977, A\&A, 55, 211

Möbius, E., Bochsler, P., Bzowski, M., et al. 2009, Sci, 326, 969

Möbius, E., Bochsler, P., Bzowski, M., et al. 2012, ApJS, 198, 11

Möbius, E., Bzowski, M., Chalov, S., et al. 2004, A\&A, 426, 897

Müller, H., Florinski, V., Heerikhuisen, J., et al. 2008, A\&A, 491, 43

Nakagawa, H., Bzowski, M., Yamazaki, A., et al. 2008, A\&A, 491, 29

Park, J., Kucharek, H., Möbius, E., et al. 2016, ApJ, 833, 130

Pryor, W., Gangopadhyay, P., Sandel, B., et al. 2008, A\&A, 28, 21

Pryor, W. R., Ajello, J. M., McComas, D. J., Witte, M., \& Tobiska, W. K. 2003, JGRA, 108, 8034

Rahmanifard, F., Schwadron, N. A., Smith, C. W., et al. 2017, ApJ, 837, 165

Richardson, J. D., Liu, Y., Wang, C., \& McComas, D. J. 2008, A\&A, 491, 1

Riedo, A., Wahlström, P., Scheer, J. A., et al. 2010, JAP, 108, 114915

Saul, L., Bzowski, M., Fuselier, S., et al. 2013, ApJ, 767, 130

Saul, L., Wurz, P., Rodriguez, D., et al. 2012, ApJS, 198, 14

Schwadron, N. A., Crew, G., Vanderspek, R., et al. 2009, SSRv, 146, 207

Schwadron, N. A., Möbius, E., Kucharek, H., et al. 2013, ApJ, 775, 86

Schwadron, N. A., Möbius, E., Leonard, T., et al. 2015, ApJS, 220, 25

Schwadron, N. A., Möbius, E., McComas, D. J., et al. 2016a, ApJ, 828, 81

Schwadron, N. A., Opher, M., Kasper, J., et al. 2016b, J. Phys. Conf. Ser., 767, 012025

Slavin, J. D., \& Frisch, P. C. 2008, A\&A, 491, 1

Sokół, J. M., \& Bzowski, M. 2014, arXiv:1411.4826v1

Sokół, J. M., Bzowski, M., Tokumaru, M., et al. 2013, SoPh, 285, 167

Sokół, J. M., Kubiak, M. A., Bzowski, M., \& Swaczyna, P. 2015, ApJS, 220, 24

Swaczyna, P., Bzowski, M., Kubiak, M. A., et al. 2015, ApJS, 220, 21

Tarnopolski, S., \& Bzowski, M. 2009, A\&A, 493, 207

Thomas, G. E. 1978, AREPS, 6, 173

Vallerga, J., Lallement, R., Lemoine, M., Dalaudier, F., \& McMullin, D. 2004, A\&A, 426, 855

Vasyliunas, V. M., \& Siscoe, G. L. 1976, JGR, 81, 1247

Witte, M. 2004, A\&A, 426, 835

Wood, B. E., Müller, H. R., Bzowski, M., et al. 2015, ApJS, 220, 31

Woods, N., Tobiska, W. K., Rottman, G. J., \& Worden, R. 2000, JGRA, 105, 27195

Woods, T. N., Prinz, D. K., Rottman, G. J., et al. 1996, JGR, 101, 9541

Wu, F. M., \& Judge, D. L. 1979, ApJ, 231, 594

Wurz, P., Saul, L., Scheer, J. A., et al. 2008, JAP, 103, 054904

Wurz, P., Schletti, R., \& Aellig, M. R. 1997, SurSc, 373, 56

Zank, G. P., Heerikhuisen, J., Wood, B. E., et al. 2013, ApJ, 763, 20 